\title{
INVESTIGACIÓN/RESEARCH
}

Recibido: 12/12/2015 ---- Aceptado: 19/02/2016 ---- Publicado: 15/03/2016

\section{FOMENTO DE LA ATENCIÓN Y LA CONDUCTA PROSOCIAL MEDIANTE LA ENSEÑANZA MUSICAL.}

Ana María Botella Nicolás ${ }^{1}$ : Universitat de València. España. ana.maria.botella@uv.es

Carolina Montesinos Boscá ${ }^{2}$ : Universitat de València. España. cmontesinos.bosca@gmail.com

\section{RESUMEN:}

El propósito principal de este artículo es investigar el grado de evolución de la capacidad de atención y de la conducta prosocial en alumnado de segundo curso de primaria mediante la enseñanza de la música. Se utilizó un diseño cuasiexperimental con diversas pruebas que se desarrollaron en 7 sesiones divididas en tres fases: pretest, intervención y post-test, en las que participaron 24 niños y niñas. Los resultados obtenidos se correspondieron con la hipótesis planteada al reflejar una evolución positiva que nos condujo a concluir que la enseñanza de la música fomenta favorablemente la atención y la conducta prosocial.

PALABRAS CLAVE: Atención - conducta prosocial - enseñanza musical investigación educativa - educación primaria

\footnotetext{
${ }^{1}$ Ana María Botella Nicolás: Profesora contratada doctora del Departamento de Didáctica de la Expresión musical, plástica y corporal de la Facultad de Magisterio de la Universitat de València.

2 Carolina Montesinos Boscá: Es Grado en Maestra en Educación Primaria, especialidad de Educación Musical por la Universitat de València.

Correo: cmontesinos.bosca@gmail.com
} 


\title{
PROMOTION OF THE ATTENTION AND THE PROSOCIAL CONDUCT BY MEANS OF THE MUSICAL EDUCATION.
}

\begin{abstract}
:
The main purpose of this paper is to investigate the degree of evolution of the capacity of attention and prosocial behavior in children in second grade by teaching music. For these purposes, a quasi-experimental design was adopted which had various tests that were developed in 7 sessions divided into three phases: pre-test, intervention and post-test, in which 24 children participated. The results obtained were consistent with the hypothesis to reflect a positive development that led us to conclude that music education promotes favorably the attention and prosocial behavior.
\end{abstract}

KEY WORDS: Attention - prosocial behavior - musical education - educational research - primary education.

\section{INTRODUCCIÓN}

El interés en la buena conducta o el fomento de la atención se encuentra muy vigente en la sociedad actual como frecuentemente puede comprobarse en los medios de comunicación, en las conversaciones casi diarias entre los padres o en las actividades de cada día de cualquier colegio. Las soluciones que suelen proponerse en los tiempos actuales para resolver el problema de las conductas no deseadas y de mejora de la atención son muy diversas. En este artículo se parte de la hipótesis de que la enseñanza de la música puede ser una buena actividad para fomentar la mejora de estas capacidades. Así pues, a través de actividades basadas en la enseñanza musical se pretende, fomentar la atención y la conducta prosocial y, por ende, conseguir un mayor rendimiento académico, mejor atención y concentración a la hora de trabajar, sin olvidar el buen clima y convivencia de los alumnos en el aula, con el fin de que aprendan a convivir en la sociedad de una manera justa e igualitaria.

El estudio se desarrolló en una clase de 24 estudiantes de primaria del Colegio de Educación Infantil y Primaria Blasco Ibáñez de la ciudad de Algemesí (ValenciaEspaña). Se observó que en la clase predominaba una gran diversidad en cuanto al rendimiento académico y unas relaciones poco amistosas. Por ello, para mejorar estas situaciones, se planteó la idea de incrementar la capacidad de atención y la concentración sin dejar de lado la conducta emocional y prosocial, estimando que la música sería una buena herramienta de intervención.

Además, se consideró importante fomentar la conducta prosocial de los alumnos a causa de que las malas relaciones interpersonales y el comportamiento antisocial pueden llevarles a retrasar su desarrollo y su crecimiento, así como ocasionar problemas de aprendizaje. Se utilizó la enseñanza de la música ya que facilita las 
relaciones humanas y permite que el individuo se adapte a su medio. Asimismo, se juzgó conveniente trabajar la capacidad de atención al considerarla esencial para un óptimo rendimiento académico. La música es un buen instrumento para mejorar la atención al ser un estímulo para enriquecer el proceso sensorial y cognitivo, además de captar el interés del alumnado. Mediante ésta se pueden llevar a cabo diversas actividades con el fin de crear hábitos de escucha y, como consecuencia, la estimulación de la atención y la memoria. La música ofrece actividades muy diversas que pueden ser tanto rítmicas, vocales, auditivas o instrumentales.

\subsection{Efectos de la música en el ser humano}

Como bien expone Betés de Toro (2000), la música ejerce su efecto a varios niveles o dimensiones del ser humano. Algunos de los efectos observados durante los últimos años son:

a) Dimensión fisiológica. La música produce respuestas de tipo fisiológico, aunque, a veces, no son fáciles de predecir. Estas respuestas pueden producirse principalmente en la respiración, en el pulso y en la presión sanguínea, en las ondas cerebrales y en la actividad muscular.

b) Dimensión emocional. Betés de Toro (2000), considera que la música tiene efectos a nivel emocional como comunicar y expresar un estado emocional (miedo, tristeza, alegría...), ayuda a la expresión de emociones profundas y provoca emociones y sentimientos.

c) Dimensión espiritual. La música ofrece paz, tranquilidad, sentimiento de felicidad, etc.

d) Dimensión cognitiva. Davis, Gfeller, y Thaut (2000) comprueban que determinadas actividades musicales pueden ayudar al desarrollo de habilidades pre-académicas y académicas y ayudar a la estimulación de funciones cognitivas como el lenguaje y la atención.

e) Dimensión social: los efectos de la música favorecen la integración social, contribuye a las relaciones sociales, facilita la cohesión grupal, invita al diálogo y comunicación con los miembros del grupo, etc.

\subsection{La atención. Aproximación conceptual.}

Tal como se ha indicado en el epígrafe precedente, entre los diferentes efectos provocados por la música en la dimensión cognitiva, se encuentra la estimulación de la capacidad de la atención. A lo largo del tiempo diversos autores han ofrecido diferentes formas de explicar en qué consiste la atención. Cabe destacar a EstévezGonzález, García-Sánchez y Junqué (1997), que consideran que el ser humano recibe constantemente estímulos sensoriales procedentes del exterior y del interior del organismo. Estos estímulos son muy abundantes y el sistema nervioso no puede procesarlos en paralelo. Por ello, la atención es un proceso que se encarga de seleccionar y organizar la percepción de los estímulos, es decir, regula la entrada de información y además está implicada en el procesamiento mismo de la información. Esta capacidad puede ser trabajada y desarrollada de forma progresiva desde la infancia hasta la edad adulta. Por tanto, se hace referencia a la definición ofrecida por Ballesteros (2002), que define la atención como la capacidad de dirigir nuestros 
recursos mentales sobre algunos aspectos del entorno, los más relevantes, o bien sobre la ejecución de determinadas acciones que consideramos más adecuadas de entre las posibles.

La atención debe ser desarrollada y trabajada en la escuela al considerarse esencial para un buen rendimiento académico. Cuando la atención se lleva a cabo intervienen diversas funciones como ejercer control sobre la capacidad cognitiva, activar el organismo ante situaciones novedosas y planificadas, determinar la dirección de la atención -la motivación- y asegurar un procesamiento perceptivo adecuado de los estímulos sensoriales más relevantes (Ballesteros, 2002).

\subsection{Tipos de atención}

Ballesteros (2002), propone la siguiente clasificación para los distintos tipos de atención existentes:

- Dependiendo del origen y naturaleza de los estímulos, la atención puede ser interna, cuando está dirigida hacía los propios procesos mentales o externa, cuando está dirigida a todo tipo de estimulación que proviene del exterior.

- Según la actitud del sujeto, la atención será voluntaria -activa-, en la cual el individuo decide el ámbito de aplicación de su capacidad atencional, o involuntaria -pasiva-, en la que el poder del estímulo es el que atrae al individuo.

En cuanto a las manifestaciones motoras, puede haber una atención abierta, acompañada de una serie de respuestas fisiológicas o una atención encubierta, la cual no es posible detectar mediante la observación. Dependiendo del interés del sujeto será atención dividida o atención selectiva, es decir, focalizada. En la atención dividida son varios los estímulos o situaciones a los que se les presta atención. En la atención selectiva el esfuerzo se dirige hacia un campo concreto en el que pueden incidir otros procesos psíquicos. Según la modalidad sensorial puede haber una atención visual, relacionada con los conceptos espaciales, o atención auditiva relacionada con parámetros temporales. Por último, se puede añadir otro tipo de atención, la atención sostenida que, como afirman Sánchez-Carpintero y Narbona (2001), permite mantener la atención focalizada en un estímulo o en una tarea durante periodos prolongados de tiempo.

\subsection{Atención y enseñanza musical}

Betés de Toro (2000) considera que la música ejerce su efecto a varios niveles o dimensiones del ser humano. Se hace referencia a la dimensión cognitiva, en la cual Davis, Gfeller, y Thaut, (2000), comprueban que determinadas actividades musicales pueden ayudar al desarrollo de habilidades pre-académicas y académicas, así como ayudar a la estimulación de funciones cognitivas como el lenguaje y la atención.

También, Senquiz (2002), hace referencia a que intelectualmente, la música desarrolla la capacidad de atención, favorece la imaginación y la creatividad. Estimula habilidades como la concentración y la memoria. Facilita el aprendizaje, ya que 
mantiene activas las neuronas cerebrales y favorece el uso de varios razonamientos a la vez, puesto que se perciben diferenciadamente elementos del sonido y se sintetizan en la captación de un mensaje integrado, armónico y lógico.

Según Soto (2002), citado en Elvira (2004), se han llevado a cabo investigaciones en el Instituto de Neurología de Londres, en las cuales se ha demostrado que el cuerpo calloso del cerebro es más grueso y desarrollado en los músicos que en el resto de personas. Esto lleva a concluir que la música aumenta las conexiones neuronales y estimula el aprendizaje y la creatividad. La música favorece la actividad cerebral completa.

En Educación Musical, cabe destacar investigaciones a través de métodos musicales que han llevado a desarrollar capacidades y hábitos de atención y concentración a partir de los sonidos. Martín (2006) menciona los sistemas metodológicos de Zoltan Kodaly y Orff-Schulwerk. Se ha descubierto que el método Kodaly mejora la concentración y atención entre otros factores. En la escuela Elemental de Clifton se aplicó el método Kodaly en recursos vocales y de movimiento y Orff en cuanto a recursos vocales e instrumentales. Se observó que ejercicios de audición de ritmos y tonos y la práctica de la fononimia ayudó a aumentar las duraciones de atención de algunos de los más lentos en aprender. Se descubrió también que estos ejercicios fomentaban la memoria tonal, aumentan la concentración y la atención. Cabe destacar que estos métodos ofrecen recursos como movimientos corporales, señales de la mano, juego y canciones folklóricas. La música es una buena herramienta para trabajar la atención, ya que los niños muestran interés en ella. Mediante ésta podemos llevar a cabo diversas actividades con el fin de crear hábitos de escucha y como consecuencia la estimulación de la atención y la memoria. Estas actividades pueden ser tanto rítmicas, como vocales, auditivas o instrumentales.

\subsection{Conducta prosocial. Aproximación conceptual}

Garaigordobil (2013) plantea dos tipos de conductas prosociales:

a) En las que hay un beneficio mutuo para las dos partes que intervienen en la relación interpersonal.

b) Las que sólo benefician a una de las partes. En este caso aparece el criterio motivacional, es decir, si las conductas prosociales tienen un fin altruista 0 no.

El comportamiento prosocial es llevado a cabo con una intención que lo define (Pérez, 2011). Este comportamiento es voluntario y los motivos pueden ser variados, es decir, pueden ser: no altruistas (egoísmo, para obtener la aprobación de los demás) y altruistas (por empatía, compasión y deseo de cuidar de los demás).

Teniendo en cuenta las características apuntadas previamente, Garaigordobil (2013, p. 44) propone una definición de conducta prosocial: "toda conducta social positiva que se realiza para beneficiar a otro con/sin motivación altruista". Esta definición incluye conductas como dar, ayudar, cooperar, compartir o consolar. Strayer (1981) expone 
cuatro tipos de actividades de comportamientos prosociales: actividades de compartir e intercambiar, cooperativas, de ayuda y empáticas.

En la intervención que nos ocupa se pretende fomentar la motivación altruista en las conductas prosociales del alumnado, por tanto, cabe volver a matizar la definición de conducta prosocial adaptada a los intereses de esta intervención. Esta definición es la expuesta por Roche-Olivar (2004, p. 39):

Aquellas acciones que benefician a otras personas, grupos (según los criterios de éstos) o metas sociales objetivamente positivas, sin que existan recompensas materiales, externas o extrínsecas, y aumentan la probabilidad de generar una reciprocidad positiva de calidad y solidaria en las relaciones interpersonales 0 sociales consecuentes, salvaguardando la identidad, creatividad e iniciativa de los individuos o grupos implicados.

\subsection{Acciones prosociales}

Roche-Olivar (2004) establece una serie de acciones prosociales que considera deben tenerse en cuenta en la educación escolar: ayuda física, servicio físico, ayuda verbal, dar, consuelo verbal, confirmación y valorización positiva del otro, escucha profunda, empatía, solidaridad, presencia positiva y unidad. Basándose en las acciones prosociales planteadas por Roche-Olivar (1995), se profundiza en el desarrollo de unas cuantas durante la intervención que se llevará a cabo, las cuales se describen a continuación:

- Ayuda. Conducta positiva que puede ser tanto no verbal como verbal que beneficia a otras personas cumpliendo un determinado objetivo. La persona que ofrece ayuda lo hace de forma desinteresada con el objetivo de beneficiar a la otra.

- Cooperación. Esta conducta no se encuentra en la lista de Roche, ya que es una forma de ayuda. En este caso, como bien afirma Garaigordobil (2003), hay un intercambio en el que los sujetos se ofrecen ayuda entre sí para obtener un beneficio en común, es decir, los miembros de un grupo dan y reciben ayuda mutuamente para llegar a un mismo fin. Éstos se relacionan de manera tal, que cada uno puede alcanzar su objetivo si, y sólo si, los otros logran alcanzar los suyos. Por tanto, hay que diferenciarla de la situación competitiva que es aquella en la que un individuo alcanza su fin si, y sólo si, los demás no logran alcanzarlos.

- Escucha profunda. Roche-Oliver (1995) se refiere a escucha como conductas de atención y respeto hacia el interlocutor en una conversación o debate. Expresa acogida paciente de lo que esta diciendo el interlocutor.

- Empatía. Es la capacidad de entender y sintonizar con los sentimientos de las otras personas. Mediante la expresión de ideas y pensamientos de uno mismo de forma voluntaria se da a entender al interlocutor que se comprenden sus pensamientos y emociones.

- Autoestima. Esta no es una acción prosocial en sí, pero es interesante tratarla como una habilidad prosocial, ya que es necesaria la toma de conciencia y el refuerzo de los aspectos positivos de uno mismo para, de esa 
forma, aprender a reconocer las emociones y adquirir un lenguaje afectivo con el fin de tener una buena relación interpersonal y llegar a la empatía.

- Confirmación y valorización positiva del otro. Valorar a otras personas de forma verbal que contribuye a aumentar la autoestima de estas. Por ejemplo: valorar las buenas conductas de otros mediante palabras de simpatía, alabanza o elogio.

\subsection{Educación socioafectiva en la escuela}

Pérez (2011) mantiene que la educación socio afectiva ha sido muy escasa y muestra un curriculum para intervenir en la escuela trabajándola. Indica que en primer lugar se debe reflexionar sobre el tipo de sociedad que se quiere y sobre el tipo de persona que se pretende que sean los alumnos y alumnas. Se sirve del "modelo sistémico" el cual tiene en cuenta la enseñanza de conocimientos reforzando el buen comportamiento, adquirir autonomía mediante estrategias y técnicas, y el establecimiento de un funcionamiento democrático en el aula. Todo esto sin olvidar la adquisición de conocimientos. Tiene en cuenta el desarrollo de la socioafectividad a partir del currículum formal y el paracurrículum. El curriculum formal ofrece estrategias para fomentar habilidades sociocognitivas (adquieren valores, pensamiento reflexivo y evolucionan desde una perspectiva egocéntrica a una mas interpersonal), afectivas (autoestima, autoimagen positiva y autoconocimiento que desemboca en la empatía) y comportamentales (autorregulación, ser respetuoso con los demás, saber escuchar reflexivamente y saber resolver problemas entre iguales mediante negociación y mediación. El paracurrículum o curriculum implícito, se encarga de que en la organización y la política educativa del centro se tenga en cuenta el cambio en las relaciones (normas, disciplina) y los métodos de trabajo para el aprendizaje de las habilidades socioafectivas. Es decir, las relaciones estimulantes de los docentes hacia los alumnos, la disciplina democrática y el aprendizaje cooperativo contando con la heterogeneidad de los individuos del grupo, recompensas y reparto de tareas.

\subsection{Conducta prosocial y música}

Betés de Toro (2000) considera que la música ejerce su efecto en varias dimensiones del ser humano y destaca la dimensión social. Explica que los efectos de la música favorecen la integración social, contribuye a las relaciones sociales, facilita la cohesión grupal, invita al diálogo y comunicación con los miembros del grupo, etc.

Desde el punto de vista de la Educación Musical, Pérez (2008, p. 191) argumenta el carácter socializador que tiene la música:

Debido a sus características, la educación musical ejerce un efecto emocional especial sobre los niños, mayor que otros fenómenos que pueden estar relacionados con la realidad (como por ejemplo la independencia del pueblo, la naturaleza, el hogar, la libertad...). Organiza y disciplina a los niños, que han de adquirirla en el seno de la comunidad, enriquece su vida emocional y les ayuda a expresarse y a establecer relaciones sociales que refuerzan el trabajo colectivo. 
La música implica una comunicación entre los hombres fortaleciendo la amistad y el entendimiento mutuo, siendo, en definitiva, un medio muy efectivo para la educación moral y estética.

En definitiva, la música se considera un buen medio para el fomento de la conducta prosocial a través de su enseñanza. Betés del Toro (2000) afirma que ésta es accesible a todos los individuos y a la comunidad. Se adapta tanto al individuo como al grupo ya que es flexible. Aporta seguridad porque tiene un orden y una estructura. Influye en la calidad de vida a través de su estética. Tiene un lenguaje simbólico-no verbal que llega a todos los individuos independientemente de las condiciones 0 estados de ánimo de estos. Y al ser multidimensional, se puede fomentar a través de ella las competencias básicas. A diferencia de la enseñanza musical, la musicoterapia utiliza la música como un medio, no como un fin, es decir, no pretende formar a músicos. Sin embargo, como es el caso de esta intervención, en algunos casos el musicoterapeuta puede considerar importante potenciar el aprendizaje de conocimientos musicales por parte del paciente con el objetivo de mejorar características socioafectivas del individuo o por otros motivos terapéuticos. Cabe destacar que se pretende fomentar la prosocialidad a través de la enseñanza musical, estos fines son tanto sociales como didácticos. Por tanto, la intervención que se llevará a cabo no es musicoterapéutica.

Fomentar las habilidades prosociales y prevenir la agresividad mediante la música ha despertado el interés de varias asociaciones que ya se han puesto en marcha, como por ejemplo:

- La aportación de Guevara (2009) sobre la fundación "Batuta" (Batuta, Sistema Nacional de Orquestas Sinfónicas Juveniles e Infantiles, 2007) que persigue el mismo objetivo que esta intervención, es decir, forma a los niños en cuanto a habilidades musicales sin dejar de lado la función socializadora. Esta fundación toma como medio la creación de grupos orquestales y el trabajo en ellos para fomentar valores tanto individuales como colectivos (autoestima, concentración, respeto, seguridad, igualdad, disciplina, pertenencia y tolerancia). Guevara (2009) también habla sobre la dirección de infancia y juventud del Ministerio de Cultura con su Plan Nacional de Música para la Convivencia (Ministerio de Cultura, 2006), el cual pretende mejorar la convivencia y construir autonomía desde el desarrollo musical.

- Por otra parte, mediante intervenciones de Musicoterapia Social como es el caso de la de Guevara (2009), también se promueve la prosocialidad y se pretende reducir el riesgo de agresividad, en este caso en niños de primaria en Bogotá, Colombia. Ésta se centra en fomentar las habilidades prosociales utilizando como medio la música pero dejando como secundario el desarrollo musical del individuo. Según Zorrillo (2004), citado por Guevara (2009), aunque en la escuela hay pocas investigaciones sobre la utilización de la musicoterapia para tratar la prosocialidad se ha demostrado que utilizar la música como medio para fomentar la prosocialidad ayuda a activar la participación y la integración 
del niño con su medio y también ayuda a estimular los repertorios básicos de aprendizaje.

- A modo de síntesis, cabe nombrar la aportación de Martín (2006), la cual destaca que tanto los objetivos de la terapia musical como los que se persiguen en la escolarización normal no son muy diferentes. En los dos se persigue favorecer la socialización, la autoestima, mejorar la atención, etc. Por ese motivo, considera aconsejable que los docentes de música compartan información sobre técnicas, conocimientos e investigaciones con el resto de docentes con el fin de utilizar la música como recurso fructífero para satisfacer las necesidades del alumnado con éxito.

\section{OBJETIVOS E HIPÓTESIS}

Los objetivos propuestos consisten en conseguir un desarrollo de la atención y de la conducta prosocial mediante la enseñanza de la música en alumnos y alumnas de segundo de primaria. Se pretende fomentar la atención del alumnado incidiendo en tres tipos de atención: sostenida, dividida y selectiva. En cuanto a la conducta prosocial, se quiere desarrollar en los niños y niñas las siguientes habilidades: empatía, confirmación y valorización positiva del otro, autoestima, cooperación, ayuda y respeto y escucha.

A modo de hipótesis, se estimó que, una vez aplicada la intervención, el alumnado habría mejorado su conducta prosocial y los diversos tipos de atención, a pesar del poco tiempo disponible. Se consideró que este período de tiempo iba a condicionar que el grado de mejoría no fuera muy relevante, pero sí el suficiente para demostrar la hipótesis en el sentido de que habría una evolución favorable por pequeña que fuera.

\section{METODOLOGÍA}

El proceso metodológico se desarrolló en cinco etapas:

$1^{\text {a }}$ Fase pre-test. Consistió en una evaluación inicial de las capacidades de atención y de conductas prosociales. Para la recogida de datos, se empleó una sesión y se utilizaron las siguientes herramientas:

- Test de evaluación de la atención selectiva

- $\quad$ Test de evaluación de atención dividida

- Test de evaluación de atención sostenida

- Cuestionario sociométrico dirigido al alumnado

- Cuestionario sociométrico dirigido educador/a

$2^{a}$ Plan de intervención. De acuerdo con la información aportada por los resultados de la fase pre-test, se plantearon los objetivos de la intervención y se planificaron las actividades. 
$3^{a}$ Intervención. Tuvo una duración de cinco sesiones. Fue utilizada la técnica de observación directa para la evaluación en cada sesión. En cada una, se llevaron a cabo actividades musicales dirigidas a fomentar la capacidad de atención y la conducta prosocial del alumnado.

$4^{a}$ Fase post-test. Tuvo una duración de una sesión y fueron utilizadas las mismas herramientas de evaluación que en la fase pre-test.

$5^{a}$ Análisis de datos e interpretación. Se analizaron los datos obtenidos en las fases post-test y pre-test de manera cuantitativa, es decir, mediante porcentajes se midió la cantidad de alumnos y alumnas que contaban con las capacidades planteadas. A continuación, se organizaron los datos en gráficos de sectores en función de los objetivos que se perseguían, se compararon también los resultados obtenidos en las dos etapas y, finalmente, se interpretaron tales resultados.

\subsection{Tipo de alumnado}

La intervención se dirigió al alumnado de la clase de segundo curso de primaria (24 niños y niñas), del CEIP Blasco Ibáñez de la ciudad de Algemesí (Valencia). El centro cuenta con un alto porcentaje de inmigrantes de procedencia magrebí y rumanos, que se caracterizan por la alta movilidad de los mismos por cuestiones laborales de sus padres. También, asisten alumnos procedentes de familias de origen sudamericano, chino, japonés y otros. En lo que concierne a las familias autóctonas se caracterizan por representar uno de los porcentajes más bajos en la escolarización de este colegio y, en buena parte, comportan problemas de desestructuración, separaciones poco amistosas, custodia de los hijos o cualquier otro problema que normalmente repercute en la conducta o rendimiento académico de los niños en la escuela.

En las observaciones realizadas se detectó un déficit en cuanto a las relaciones prosociales y al rendimiento académico. Se observaron frecuentes disputas y agresiones entre ellos, burlas hacia los alumnos más rezagados, falta de colaboración y ayudas, si bien algunos de ellos mostraban una fuerte competitividad. También faltas de respeto no sólo hacia los compañeros, sino hacia los maestros, puesto que algunos de ellos apenas escuchaban las explicaciones de la maestra y continuamente alteraban el ritmo de la clase hablando con otros compañeros.

\subsection{Instrumentos de evaluación: pre-test y post-test.}

Para evaluar la evolución de la capacidad de la atención y la conducta prosocial se desarrolló un proceso evaluativo en fase pre-test y post-test. El proceso de la fase pre-test consistió en cumplimentar, en una sesión de 45 minutos, diversos test antes del comienzo de la intervención, con la idea de observar el nivel inicial de dichas capacidades, detectar sus déficit y planificar la intervención adecuada para desarrollarlas. Posteriormente, una vez aplicada la intervención, los alumnos y alumnas, en la fase post-test, cumplimentaron los mismos test presentados en la fase anterior con el fin de comprobar la evolución. 
Los test se dividieron en dos secciones en las que se contemplaba, por una parte, las pruebas relacionadas con la capacidad de atención y, por otra, los cuestionarios que evaluaban la conducta prosocial. En cuanto a los test relativos a la capacidad de atención, se tuvo en cuenta los tres principales tipos de atención: atención dividida, atención sostenida y atención selectiva, los cuales se evaluaron mediante actividades de discriminación auditiva del timbre y de la intensidad. Para la evaluación de la conducta prosocial, se tuvo en cuenta los parámetros de autoestima, empatía, cooperación, ayuda, respeto y escucha mediante dos cuestionarios, uno que fue cumplimentado por la docente y otro por el alumnado.

La técnica de observación directa también se utilizó como instrumento de evaluación con lo cual se pudo detectar el déficit del alumnado tanto a nivel de atención como de prosocialidad. Además durante las fases evaluativas de pre-test y post-test y durante la intervención, se usó de herramienta para analizar en cada una de las actividades el grado de placer del alumnado, la participación, el acatamiento de las reglas, el clima de grupo, la escucha, las interacciones de ayuda y cooperación, es decir, las acciones prosociales que se pretendieron fomentar, así como los elementos positivos y negativos de la sesión.

\subsection{Diseño y procedimiento del programa de intervención}

La intervención constó de tres fases: La fase inicial pre-test (1 sesión), la fase de intervención ( 5 sesiones) y la fase post-test (1 sesión). En total 7 sesiones repartidas en dos por semana y con una duración de 45 minutos. La intervención se diseñó a partir de los resultados observados en la fase pre-test. Se elaboraron una serie de actividades para potenciar conjuntamente tanto las habilidades prosociales como la capacidad de atención en las cuales se observó déficit en la fase pre-test. Se establecieron los objetivos ya mencionados y se planificaron las sesiones con sus respectivas actividades en la fase de intervención. Cada sesión se organizó con el fin de que contuviera una o dos actividades y un debate de reflexión final. Las actividades se programaron en base a la información recogida en los artículos, documentos y tesis doctorales que relacionan el desarrollo musical con el fomento de las capacidades propuestas. Cabe destacar que aunque el comportamiento social está muy relacionado con la atención, las intervenciones que se estudiaron las fomentaban de forma separada. No obstante, al observar estas dos carencias en el alumnado se pretendió encontrar una manera de fomentarlas de forma conjunta y a través de actividades musicales.

Para fomentar la prosocialidad las actividades que se trabajaron en la intervención fueron de carácter cooperativo, por equipos, de ayuda entre compañeros, empáticas como la del alumno en el papel de tutor, de fomento de la autoestima, mediante la elección de representantes y de valoración positiva del otro al tener en cuenta el esfuerzo de sus compañeros al realizar las actividades. Al mismo tiempo, en estas actividades se trabajó mediante la música la atención selectiva, al centrar su atención en un determinado estímulo, la atención dividida en actividades cuyo objetivo era que se prestara atención a varios estímulos a la vez y la atención sostenida, mediante tareas que requerían una atención duradera en el tiempo para conseguir su éxito. 
La intervención fue planificada para que todos los objetivos buscados fueran trabajados, no obstante, era inevitable trabajar unos más que otros. Es decir, en cuanto a la capacidad de atención, la atención sostenida se trabajó en 5 sesiones, la atención dividida en 3 y la atención selectiva en 2 . En cuanto a las habilidades prosociales, la cooperación se trabajó en 4 sesiones, la autoestima, la escucha y el respeto en 3 y la ayuda y la valoración positiva del otro en 1 sesión. Posteriormente, se observó que esto también influyó en los resultados obtenidos en la fase post-test.

\section{DISCUSIÓN}

\subsection{Análisis de los datos}

En primer lugar, se analizaron los resultados obtenidos en la fase pre-test con el fin de planificar las sesiones de la intervención considerando las características que presentaban cada alumno y alumna. En segundo lugar, para comprobar la eficacia de la intervención aplicada, se llevó a cabo un análisis cuantitativo con el programa informático SPSS versión 19.0, es decir, se agruparon los datos de cada fase en parámetros, se transformaron en porcentajes, se representaron en gráficos de sectores y se compararon las fases pre-test y post-test entre ellas.

En cuanto a los tres test que evalúan la atención, los datos se recogieron agrupados en parámetros. Es decir, se clasificaba al alumnado dependiendo de la puntuación obtenida en los test como "alumnos con falta de capacidad de atención", aquellos cuya puntuación obtenida fuera de 0 a 4,99; como "alumnos con atención adquirida" a aquellos que tuvieran una puntuación de entre 5 y 8,99 puntos y; como alumnos con "máxima capacidad de atención" a los que puntuaran de 9 a 10 puntos. Una vez agrupados, se calcularon las cantidades de cada tipo de alumnos en porcentajes y se representaron en gráficos de sectores para observar qué sectores predominaban en la clase con el fin de cuantificar el nivel de la clase en lo referente a esta capacidad. Una vez hecho esto en cada fase por separado, se compararon las dos fases para observar si había habido modificaciones en esta capacidad después de aplicar la intervención.

En el cuestionario dirigido al docente, el análisis de los datos se llevó a cabo teniendo en cuenta el comportamiento que presentaba cada alumno desde el punto de vista del profesor. Es decir, si un alumno en el cuestionario presentaba más cantidad de $\mathrm{X}$ en "Nunca" se le clasificó como alumno con falta de comportamiento prosocial; el alumno que tuvo mayor cantidad de $\mathrm{X}$ en "alguna vez" se le clasificó como alumno con poco comportamiento prosocial y el alumno que obtuvo mayor cantidad de $\mathrm{X}$ en "Casi siempre" se le clasificó como alumno con comportamiento prosocial alto. Con esto se pretendió medir la cantidad de alumnos existentes en la clase con nivel comportamiento prosocial alto, con poco nivel y sin comportamiento prosocial. Una vez analizada la fase pre-test y la post-test se compararon entre ellas para observar la evolución.

En lo referente al análisis de los cuestionarios dirigidos al alumnado, se agrupó en "alumnado prosocial" a todos aquellos alumnos nombrados en el apartado de felicitación. Como alumnado "no prosocial" a los alumnos y alumnas mencionados por sus compañeros en el apartado de mejorar conducta $y$, por otra parte, se tuvo en 
cuenta la cantidad de alumnos que no se mencionaron. Con esto se comprobaba si el alumnado consideraba que en la clase predominaban los alumnos con comportamiento prosocial o sin él. Posteriormente, se compararon las dos fases con el fin de averiguar si hubo modificaciones en la fase post-test.

Una vez analizados los dos cuestionarios, el de la docente y el del alumnado, por separado, se compararon entre ellos en cada fase. Es decir, el cuestionario que la docente realizó en la fase pre-test se comparó con el del alumnado de esa misma fase y de la misma forma con los de la fase post-test. El proceso de comparación consistió en analizar las características que atribuía la maestra al alumnado que sus compañeros mencionaban como prosociales, no prosociales y no mencionados con el fin de observar si había una relación acertada entre las opiniones del alumnado y de la propia docente. Cabe añadir que los parámetros del cuestionario de la maestra se correspondían con los del cuestionario del alumnado de la siguiente manera: alumnos con comportamiento prosocial alto se identificaba con alumnos prosociales, alumnos con falta de prosocialidad se correspondía con alumnos no prosociales y alumnos con poca prosocialidad era un nivel intermedio pero se identificaba más como alumnado no prosocial.

En último lugar, se analizaron los resultados obtenidos de la observación directa de una manera cualitativa, es decir, la evolución que había tenido el alumnado en cuanto a su comportamiento prosocial durante la aplicación de las sesiones y después se comparó la visión de los dos cuestionarios con estos resultados para comprobar si coincidían ${ }^{3}$.

\subsection{Pruebas evaluativas de la capacidad de atención Capacidad de atención dividida}

Los resultados del estudio del test de la atención dividida fueron los que se observan en las figuras 1 y 2 :

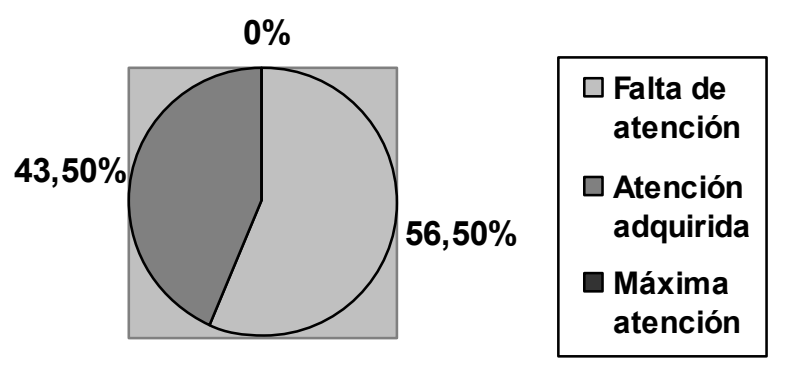

\footnotetext{
${ }^{3}$ Como aclaración previa, conviene indicar que de los 24 alumnos matriculados, en la fase pre-test participaron 23 y en la fase post-test, 20, debido a las faltas de asistencia a clase. En cuanto a los cuestionarios sociológicos fueron cumplimentados teniendo en cuenta a la totalidad de los alumnos y alumnas.
} 


\section{Gráfico 1: Resultados del análisis de la capacidad de atención dividida en la fase pre-test}

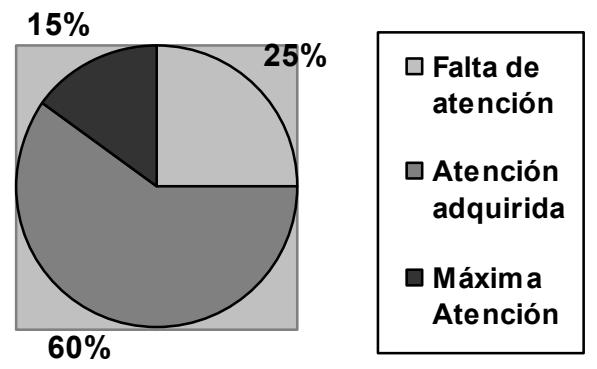

\section{Gráfico 2: Resultados del análisis de la capacidad de atención dividida en la fase post-test}

En la comparación de los gráficos anteriores, se puede observar una evolución bastante positiva en cuanto a la mejora de la capacidad de la atención dividida. En la fase pre-test el porcentaje de alumnos que predomina es el que tiene falta de atención dividida (56,50\%), el segundo sector predominante es el alumnado con un nivel de atención dividida adquirido (43,50\%) y el sector de alumnos con máxima atención es inexistente.

En cuanto a los resultados de la fase post-test, se observan considerables diferencias a favor de una evolución positiva de esta capacidad en los alumnos; el porcentaje mayoritario pasa a ser el del alumnado con la atención dividida adquirida en una cuantía del $60 \%$ que supone un incremento favorable del $16,50 \%$ sobre la fase anterior; el porcentaje del alumnado con falta de atención dividida se reduce al $25 \%$, que representa comparativamente una mejoría de $31,50 \%$, y, finalmente, el aumento del alumnado con máxima atención dividida refleja un $15 \%$ de mejora.

\section{Capacidad de atención sostenida}

En cuanto a la prueba de atención sostenida, los resultados fueron los siguientes:
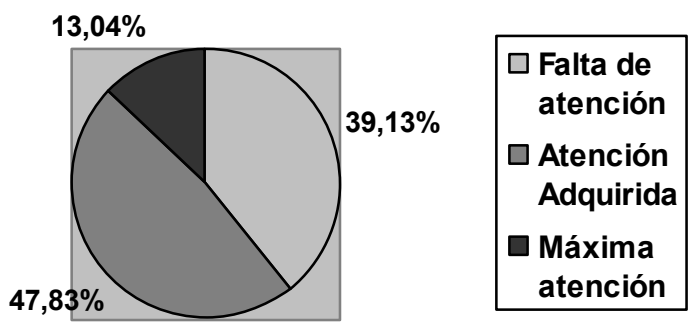


\section{Gráfico 3: Resultados del análisis de la capacidad de atención sostenida en}

la fase pre-test

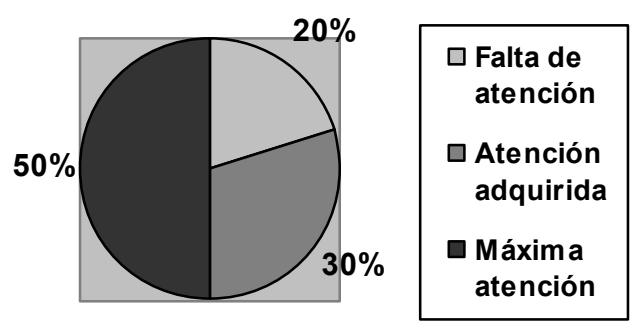

\section{Gráfico 4: Resultados del análisis de la capacidad de atención sostenida en la fase post-test}

En esta prueba se observa una mejora considerable de una fase a otra. En la fase pretest había un gran porcentaje de alumnos con falta de atención sostenida $(39,13 \%)$, el sector predominante era el alumnado con atención sostenida adquirida $(47,83 \%)$ y el sector minoritario, el alumnado con la máxima atención sostenida (13,04\%). No obstante, en la fase post-test el sector mayoritario fue el alumnado con máxima atención sostenida $(50 \%)$, con un aumento del $36,96 \%$, quedando en segundo lugar el sector de alumnado con atención sostenida adquirida (30\%), con una disminución del $17,83 \%$ y el sector minoritario fue el alumnado con falta de atención sostenida ( $20 \%$ al reducirse en un $19,13 \%)$.

\section{Capacidad de atención selectiva.}

Los resultados obtenidos en la prueba de atención selectiva fueron:

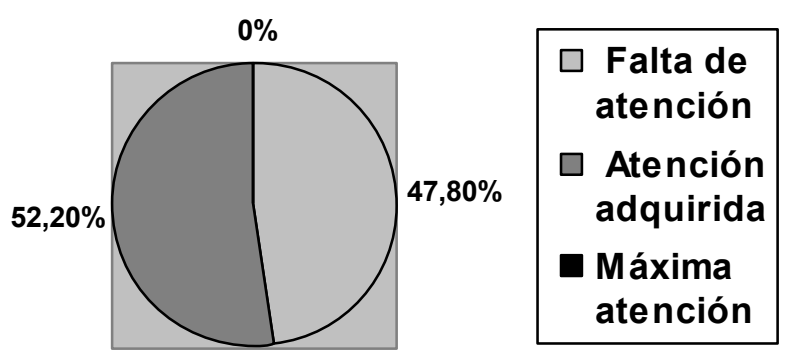

Gráfico 5: Resultados del análisis de la capacidad de atención selectiva en la fase pre-test

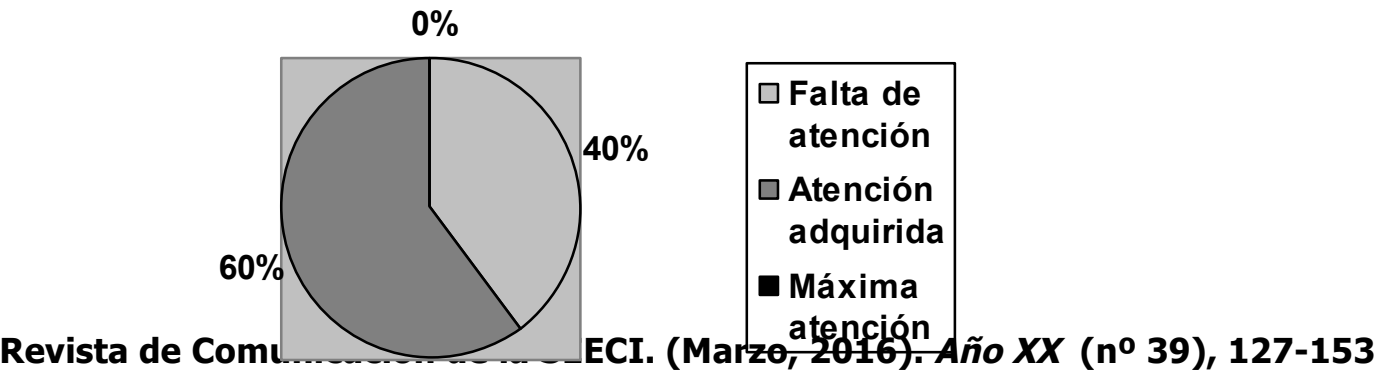


Gráfico 6: Resultados del análisis de la capacidad de atención selectiva en la fase post-test

En esta prueba, también se observa una evolución favorable de la capacidad de atención selectiva del alumnado, aunque en menor medida. La máxima atención selectiva es inexistente en las dos fases, la falta de atención adquirida señala una diferencia menor del $7,80 \%$ en la fase post-test y un aumento de la atención adquirida del $7,80 \%$.

Pruebas evaluativas del comportamiento prosocial del alumnado Cuestionario sociométrico realizado por el educador/a

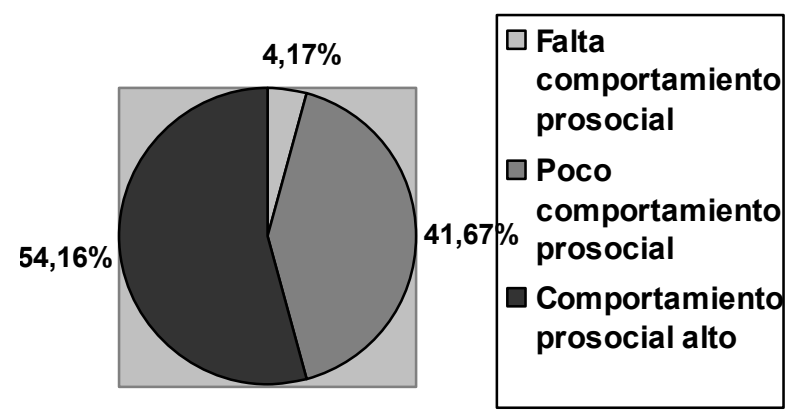

Gráfico 7: Resultados del análisis del comportamiento prosocial desde el punto de visto del educador/a en la fase pre-test

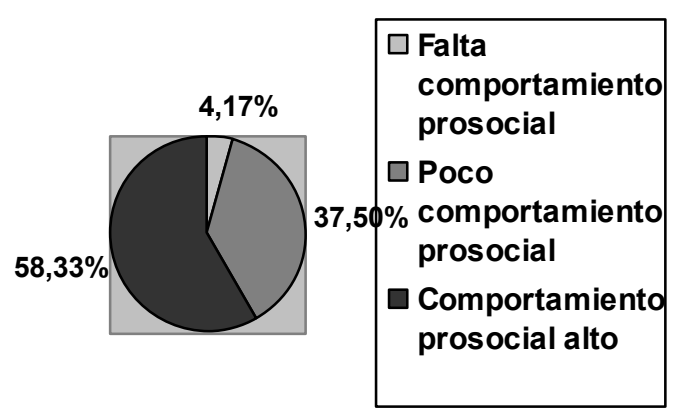




\section{Gráfico 8: Resultados del análisis del comportamiento prosocial desde el punto de visto del educador/a en la fase post-test}

En los gráficos se puede observar que en la fase pre-test predomina el comportamiento prosocial alto $(54,16 \%)$, seguido por el comportamiento poco prosocial $(41,67 \%)$ y como sector minoritario la falta de comportamiento prosocial $(4,17 \%)$. En la fase post-test hay un aumento del alumnado con conducta prosocial alto en un porcentaje del $4,17 \%$, el cual se mantiene como sector mayoritario, se reduce en el mismo porcentaje el sector con poca prosocialidad y se mantiene el porcentaje del alumnado con falta de prosocialidad.

\section{Sociograma dirigido a los alumnos}

En el cuestionario dirigido al alumnado, se analizó la cantidad de alumnado calificado como prosocial, el considerado no prosocial y los no mencionados:

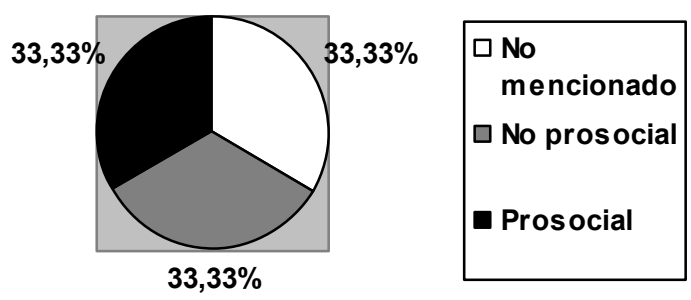

\section{Gráfico 9: Resultados del análisis del comportamiento prosocial desde el punto de vista del alumnado en la fase pre-test}

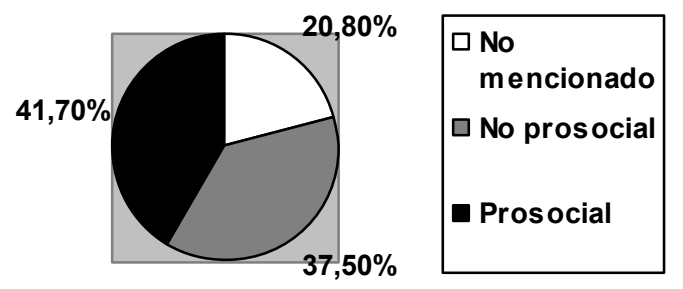

\section{Gráfico 10: Resultados del análisis del comportamiento prosocial desde el punto de visto del educador/a en la fase post-test}

Se observa una evolución de mejora relativa. En la fase pre-test todos los sectores indican el mismo porcentaje (33,33\%). En la fase post-test se reduce el sector de no $(12,53 \%)$, aumenta el sector no prosocial $(4,17 \%)$, y también se incrementa el sector prosocial en un $8,37 \%$ quedando como el sector mayoritario. 


\section{Relación entre ambos sociogramas}

Los resultados que reflejan la comparación entre los cuestionarios cumplimentados por los alumnos y su profesora fueron los siguientes:

\section{En la fase pre-test}

A) El alumnado mencionado por sus compañeros como alumnos con conductas prosociales, reúnen las siguientes características según la docente:
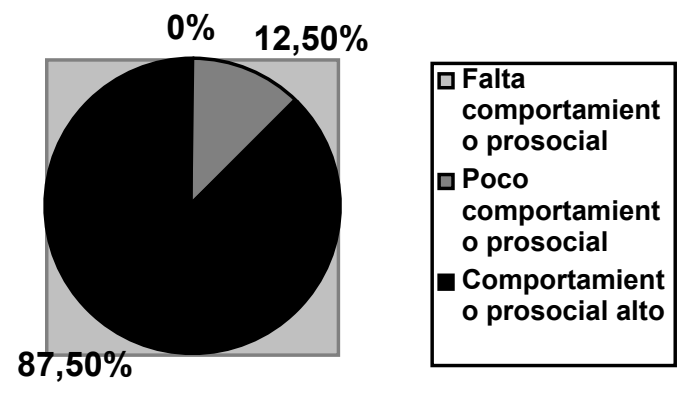

\section{Gráfico 11: Alumnado evaluado por sus compañeros como prosocial analizado desde el punto de visto del docente en la fase pre-test}

Los alumnos y alumnas mencionados por el alumnado como prosociales son identificados por la maestra como alumnos con conductas mayoritarias prosociales, menos un pequeño porcentaje que destaca en sus conductas poco prosociales. Analizando con más profundidad el cuestionario de la docente, observamos que algunos son poco prosociales en ofrecer ayuda, en mostrar empatía con la docente y en el respeto a los compañeros. Cabe destacar que todos tienen la característica común de poder trabajar en pequeño grupo.

B) En lo referente al alumnado mencionado por sus compañeros como alumnado no prosocial, resaltar que en el cuestionario de la docente estos alumnos son mencionados como alumnos prosociales altos, alumnos con poco comportamiento prosocial y alumnos con falta de comportamiento prosocial en estos porcentajes: 

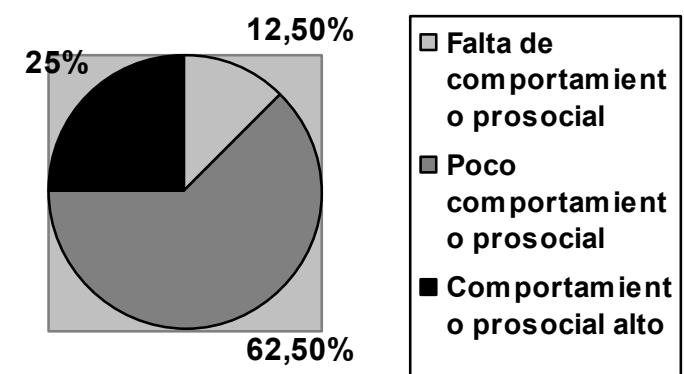

\section{Gráfico 12: Alumnado evaluado por sus compañeros como no prosocial analizado desde el punto de visto del docente en la fase pre-test}

Las características comunes de este alumnado es que no respetan a la docente ni a los demás compañeros y, sobretodo, son poco capaces de trabajar en pequeño grupo.

C) Por último, en lo referente al alumnado no mencionado por sus compañeros, cabe hacer referencia que en el cuestionario de la docente se corresponden con los siguientes sectores:

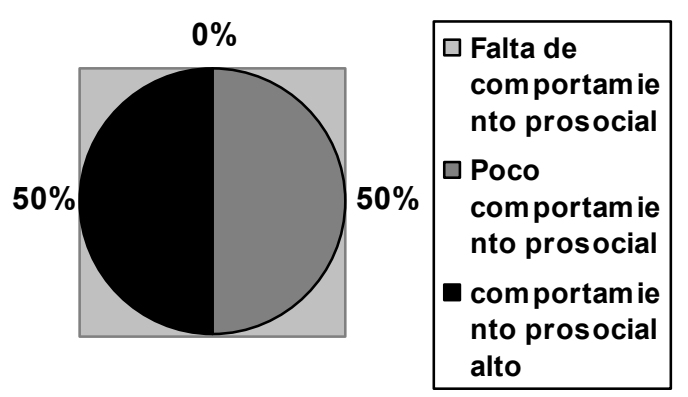

Gráfico 13: Alumnado no mencionado por sus compañeros analizado en cuanto a su conducta prosocial desde el punto de visa del docente en la fase pre-test

Este alumnado destaca por tener conductas mayoritarias prosociales o poco prosociales.

\section{En la fase post-test}

A) Por otra parte, en lo referente a la fase post-test, el alumnado considerado por sus compañeros como alumnado prosocial es clasificado por la docente de la siguiente manera: 

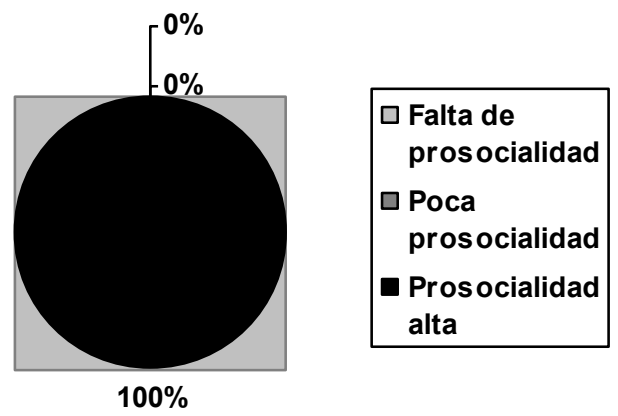

\section{Gráfico 14: Alumnado considerado prosocial desde el punto de vista del docente en la fase post-test}

Se puede observar que la opinión del alumnado se corresponde completamente con la opinión de la docente. Estos alumnos tienen en común que ayudan a los compañeros, respetan y escuchan al docente y pueden trabajar en equipo.

B) En cuanto al alumnado que es considerado por sus compañeros como no prosocial, la docente los clasifica tal y como se aprecia en el gráfico 15:
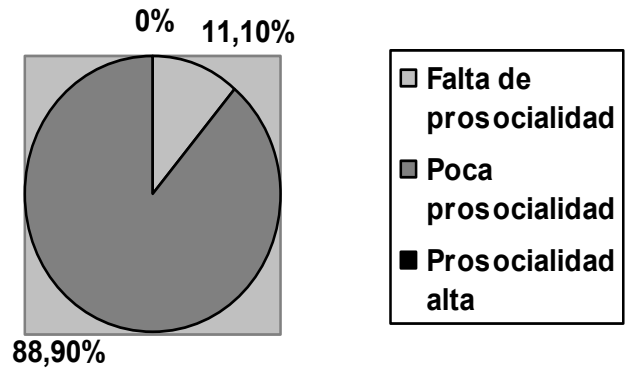

\section{Gráfico 15: Alumnado considerado como no prosocial por sus compañeros analizado desde el punto de vista del docente en la fase post- test}

Se observa que la docente identifica a los alumnos mencionados por sus compañeros como alumnos con falta de prosocialidad en mayor medida y como alumnos con poca prosocialidad en menor medida. Cabe destacar que tienen en común características como la falta de respeto y escucha a la docente y compañeros y que les resulta difícil trabajar en grupo.

C) En lo referente a los compañeros y compañeras no mencionados por el alumnado cabe destacar que la docente los identifica como alumnado con prosocialidad alta y con poca prosocialidad en las siguientes medidas: 


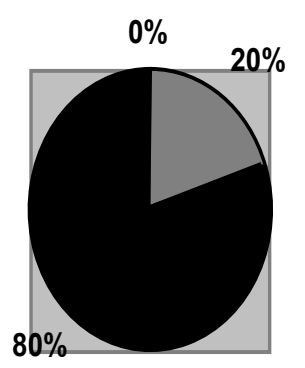

G Falta de prosocialidad

口oca prosocialidad

Q Prosocialidad alta

\section{Gráfico 16: Alumnado no mencionado por sus compañeros analizado en cuanto a su comportamiento prosocial desde el punto de vista del docente en la fase post-test}

\section{CONCLUSIONES}

\subsection{Capacidad de atención}

Respecto a los resultados de la capacidad de atención del alumnado, en concreto al tipo de atención sostenida, la mayoría de ellos partieron de un nivel adquirido del $47,83 \%$; también hubo un alto porcentaje de alumnos con falta de este tipo de atención del $39,13 \%$, y un pequeño porcentaje con una capacidad máxima de atención sostenida del $13,04 \%$. Cabe destacar que este tipo de atención fue la segunda capacidad que más se desarrolló de las tres, ya que en la fase post-test, hubo un aumento del $36,96 \%$ del alumnado con capacidad máxima de atención siendo éste el sector mayoritario de la clase, disminuyendo un $19,13 \%$ el alumnado con falta de atención sostenida. También disminuyó en menor medida el porcentaje de alumnos con capacidad de atención sostenida adquirida quedando el segundo sector mayoritario. Por tanto, el alumnado que mejoró su capacidad de atención sostenida fue un $36,96 \%$ de la clase quedándose en un $20 \%$ el alumnado con falta de atención el más reducido de los tres tipos de atención trabajados.

En lo que concierne a la capacidad de atención selectiva, destacar que, en un principio, la mejora se notó algo menos. En la fase pre-test, predominó el alumnado con atención selectiva adquirida pero sólo en un $4,4 \%$ más que el alumnado con falta de esta atención y el alumnado con la máxima capacidad de atención selectiva fue inexistente. Se muestra su mejora en que en la fase post-test aumentó en un $7,8 \%$ el alumnado con capacidad de atención selectiva adquirida pero nadie logró alcanzar el nivel de máxima atención selectiva.

Consecuentemente, en este caso se observó una mejora muy notable de la capacidad de atención selectiva al producirse un aumento del $40 \%$ del alumnado con capacidad 
de atención selectiva máxima. Se redujo un $32,2 \%$ el porcentaje del alumnado con atención selectiva adquirida y en menor medida el alumnado con falta de esta capacidad en un 7,8\%, el cual pasó a tener el mismo valor que el porcentaje de alumnos con máximas capacidades. El alumnado con atención adquirida pasó a ser el sector minoritario. Por tanto, un $40 \%$ del alumnado de la clase mejoró en el desarrollo de esta capacidad cuando estaba ya adquirida en su mayoría, siendo esta la mejora más notable de las tres tipos de atención trabajados. Por otra parte, el porcentaje del alumnado con falta de atención se mantuvo como el más alto de los tres tipos de capacidad de atención trabajados en la fase post-test (40\%) y se observó una mejora poco relevante en este.

Por último, en lo referente a la atención dividida, se detectó que en la fase pre-test el alumnado partió de un nivel bastante bajo, ya que el porcentaje más alto fue el de la falta de atención aunque con una diferencia del $13 \%$ en comparación con el porcentaje del alumnado con la capacidad de atención dividida adquirida, siendo inexistente el porcentaje de alumnado con máxima atención dividida. Cabe destacar que el porcentaje de alumnado con falta de este tipo de atención en la fase pre-test es el más alto de los tres tipos de atención estudiados; la fase post-test nos mostró un buen desarrollo al reflejar una disminución del $31,5 \%$ en el alumnado con falta de capacidad; con esta disminución se mostró un aumento de los porcentajes de alumnado con la capacidad adquirida y con la capacidad máxima, no obstante, el alumnado con capacidad adquirida fue el que más aumentó pero con una diferencia de un $1,5 \%$, casi irrelevante en lo que concierne a la capacidad máxima, resultando así mayoritario el alumnado clasificado con la capacidad de atención dividida adquirida. Por tanto, mejoró la capacidad de atención dividida en un $31,5 \%$ de la clase, o sea, la mejora más pequeña de los tres tipos de atención estudiados.

A modo de síntesis, la intervención aplicada al alumnado contribuyó a la mejora de los tres tipos de atención. Basándonos en las últimas reflexiones y modificaciones, la capacidad de atención selectiva fue la capacidad en la cual mayor mejoría se observó en los alumnos al reflejar el $40 \%$ de la clase, no obstante, aunque se redujo la falta de esta capacidad, el alumnado con falta de ésta fue el porcentaje más alto de los tres tipos de atención estudiados (40\%); en segundo lugar, el 36,96\% del alumnado mejoró su capacidad de atención sostenida, quedando el porcentaje del alumnado con falta de atención el más reducido de los tres tipos de atención trabajados con un $20 \%$ y el más alto de alumnado con la capacidad de atención máxima en un $50 \%$ y; en tercer lugar, en el desarrollo de la atención dividida, el alumnado que mejoró esta capacidad fue el $31,5 \%$ de la clase. Por último, cabe destacar que, actualmente, en cuanto al porcentaje de alumnado con máxima capacidad de atención, la capacidad de atención sostenida es la mayoritaria en el alumnado (50\%); en segundo lugar, la atención selectiva (40\%) y; en último lugar, la atención dividida (15\%). No obstante, si se tiene en cuenta al alumnado que ha adquirido estas capacidades ya sea de forma básica o máxima, la atención sostenida es la más adquirida por el alumnado (80\%); en segundo lugar, quedaría la atención dividida $(75 \%)$ y; en último lugar, la atención selectiva (60\%). Considero que en estos resultados ha influido la forma en que se ha trabajado cada tipo de atención, ya que, en 5 sesiones se trabajó la atención sostenida, en 3 la atención dividida y en 2 la atención selectiva, lo cual ha provocado 
que cuanto más se ha trabajado cada tipo de atención, más se ha reducido el alumnado con falta de estas capacidades.

\subsection{Comportamiento prosocial}

En cuanto al comportamiento prosocial del alumnado, desde la perspectiva de la docente, se observa que hubo una pequeña evolución favorable al aplicar la intervención, lo cual se muestra en los resultados obtenidos del cuestionario de la fase post-test en el cual hay un aumento del porcentaje del alumnado con conducta prosocial alta en un $4,17 \%$ con respecto a la fase pre-test, representando en las dos fases el sector mayoritario. Se reduce la cantidad de alumnos en el tramo de poca prosocialidad y se mantiene el porcentaje del alumnado con falta de prosocialidad. Apenas tiene relevancia el porcentaje del alumnado que desarrolla su conducta prosocial al suponer sólo un aumento del 4,17\%. En la fase post-test, aunque el sector mayoritario sigue siendo el alumnado con alta prosocialidad, los alumnos situados en el tramo de poca o falta de ella siguen siendo un sector importante de la clase puesto que sólo se diferencian de los alumnos con alta prosocialidad en un $16,66 \%$ menos.

En lo que respecta al cuestionario dirigido al alumnado, mientras que en la fase pretest todos los sectores tenían el mismo porcentaje, en la fase post-test el mayoritario pasó a ser el alumnado con conductas prosociales con un aumento del 8,73\% aunque sigue habiendo un gran sector de la clase considerado como no prosocial que sólo se diferencia del porcentaje del alumnado prosocial en un 4,17\%. El alumnado no empeoró su conducta, es más, pensamos que la mejoró. No obstante, se mencionaron alumnos en la fase pos-test que en la pre-test pasaron desapercibidos y por ese motivo aumentaron tanto los alumnos no prosociales como los prosociales en esta última fase.

Al comparar el cuestionario dirigido a la docente y el cuestionario dirigido al alumnado se observó que existe bastante relación entre uno y otro, tanto en la fase pre-test como en la post-test, pero en la fase post-test los resultados fueron más parecidos. Ambos coincidieron en que el alumnado prosocial es el mayoritario y en la contemplación de una mejora en las conductas. En la fase pre-test, el alumnado mencionó alumnos prosociales a los que la maestra también calificó de igual manera aunque en menor porcentaje, aparecieron los poco prosociales. En cuanto a los alumnos mencionados como no prosociales, la maestra los caracterizó en la mayoría como poco prosociales, en menor porcentaje como prosociales altos y como minoritario los no prosociales; los no mencionados pasaron desapercibidos y fueron tanto prosociales como poco prosociales.

Por todo ello, debemos destacar que en la fase pre-test el alumnado y la maestra no tienen la misma opinión de los individuos de la clase, aunque se aproximan bastante. La maestra considera que hay más alumnos prosociales de lo que el alumnado percibe de sus compañeros. En la fase post-test, se observó una mayor aproximación entre la visión de la docente y del alumnado. El alumnado y la maestra coincidieron en los calificados como prosociales. En cuanto a los alumnos mencionados como no 
prosociales, en menor medida la docente los calificó como no prosociales y en mayor medida como poco prosociales. El gráfico de no mencionados nos mostró que pasaron desapercibidos alumnos que la docente consideró prosociales altos en su mayoría y, también, poco prosociales. En consecuencia, debemos resumir que la maestra y el alumnado ofrecen una visión bastante aproximada de los comportamientos de los individuos de la clase, pero, ésta observa una mayor prosocialidad. En cuanto a las características del alumnado, cabe destacar que el alumnado considera prosociales a los compañeros que la maestra considera como capaces de trabajar en grupo, escuchar y respetar a la maestra, y como no prosociales a aquellos menos capaces de trabajar en grupo y poco respetuosos con la maestra. Los alumnos dieron menor importancia en la fase pre-test al ofrecer ayuda a los compañeros o ser respetuosos entre ellos, por eso, mencionaron en algunos casos como prosociales a compañeros que carecen de estas habilidades.

\subsection{Observación directa}

La observación directa de las actividades de esta intervención permitió detectar cómo el alumnado fue mejorando sus conductas prosociales con respecto al docente y a los propios compañeros. Se observa en la fase pre-test una conducta bastante negativa por parte del alumnado en cuanto a la falta de escucha y respeto a la docente, no obstante se apreciaron interacciones de ayuda entre ellos. En la primera sesión de la intervención, tuvo lugar una importante mejora en su atención y respeto a la docente, con un clima de grupo muy organizado y pacífico, quizás debido a la novedad de las actividades que despertó su interés. En la segunda sesión, el comportamiento prosocial vuelve a disminuir, ya que algunos de ellos no escuchaban a la maestra aunque la mayoría mostraban interés. También, aparece el rechazo a formar parte de un grupo con determinados compañeros pero se mantiene la conducta de ayuda en otros. La tercera, cuarta y quinta sesión incluyendo la sesión post-test fueron muy positivas, en las que únicamente se observaron comportamientos prosociales al escuchar y respetar al docente y a sus compañeros, prestándose conductas de ayuda y cooperando. Por tanto, relacionando las observaciones directas con la de la docente y los alumnos de la clase, se puede demostrar un aumento de la conducta prosocial del alumnado.

\subsection{Consideraciones finales}

Finalmente, podemos concluir que en la investigación realizada se pudo comprobar la hipótesis inicial de que la enseñanza musical contribuye en el ámbito de la educación a la mejora de la atención y de la conducta prosocial. No obstante, no se desarrollaron en la misma intensidad, puesto que la capacidad de la atención progresó en mayor medida y el comportamiento prosocial de forma menos notable. En cuanto a la capacidad de atención, se observó que en su mejora influyó, el tiempo de trabajo dedicado obteniendo mejores resultados en los tipos de atención más trabajados. En relación a la prosocialidad aunque, según la maestra y el alumnado, la cantidad de alumnos que mostraron mejoría es poco relevante, durante la intervención se detectaron importantes avances, sobre todo en el respeto, la escucha, la ayuda y la cooperación y, en menor cantidad en la autoestima, en la empatía y en la valoración 
positiva del otro. Debemos resaltar que los porcentajes de mejora no superaron el $40 \%$, porcentaje nada despreciable teniendo en cuenta que la duración de la intervención fue muy breve. Esto nos conduce a ser optimistas en la aplicación de la enseñanza musical en la escuela ya que simplemente es cuestión de tiempo para lograr resultados positivos en cuanto a la capacidad de atención y en la conducta prosocial en el alumnado.

\section{REFERENCIAS}

Ballesteros, S. (2002). Psicología General. Un enfoque cognitivo para el siglo XXI. Madrid: Editorial Universitas.

Betés de Toro, M. (2000). Fundamentos de musicoterapia. Madrid: Ediciones Morata, 2000.

Campos, E., Caño, M., Carrión, D., Gallardo, C., González, I. et al. (2008). Aplicaciones Didácticas de la Musicoterapia en el Aula de Primaria. Málaga: Ediciones Guillermo Castilla.

Davis, W. B., Thaut, M. H., \& Gfeller, K. E. (2000). Introducción a la musicoterapia: teoría y práctica. Barcelona: Boileau.

Elvira, M. A. (2004). Propuesta Pedagógica para la utilización de la musicoterapia en la atención de niños que presentan dificultades de aprendizaje. (Tesis de Grado). Universidad Nacional Abierta, Caracas.

Estévez-González, A., García-Sánchez, C., \& Junqué, C. (1989). La atención: una compleja función cerebral. Revista de neurología, 25 (148), 1989-1997.

Garaigordobil, M. (2003). Diseño y evaluación de un programa de intervención socioemocional para promover la conducta prosocial y prevenir la violencia. Primer premio nacional de investigación educativa. España: Centro de Investigación y Documentación Educativa (C.I.D.E).

Guevara, M. P. (2009). Intervención musicoterapéutica para promover la prosocialidad y reducir el riesgo de agresividad en niños de básica primaria y preescolar en Bogotá, Colombia. International Journal of Psychological Research, 2 (2), 128-136.

Lacárcel, J. (2003). Psicología de la música y emoción musical. Revista Educatio, 20$21,213-226$.

Martín, E. (2006). Aptitudes musicales y atención en niños entre diez y doce años. (Tesis inédita de doctorado). Departamento de psicología y sociología de la educación, Universidad de Extremadura. 
Pérez, S. (2008). El ritmo: una herramienta para la integración social. Ensayos: Revista de la Facultad de Educación de Albacete, 23, 189-198.

Pérez, D. (2011). Programa de Intervención Musicoterapéutica breve para el Incremento de la Prosocialidad en centros de Educación Secundaria, dentro del Programa de Acción Tutorial. (Tesina de Máster). Máster en Musicoterapia ISEP, Valencia.

Roche-Olivae, R. (1995). Psicología y educación para la prosocialidad. Bellaterra: Servicio de publicaciones Universidad Autónoma de Barcelona.

Roche-Olivar, R. (2004). Inteligencia prosocial: educación de las emociones y valores. Bellaterra: Servicio de publicaciones Universidad Autónoma de Barcelona.

Sánchez-Carpintero, R., \& Narbona, J. (2001). Revisión conceptual del sistema ejecutivo y su estudio en el niño con trastorno por déficit de atención e hiperactividad. Revista de Neurología, 33, 47-53.

Senquiz, A. (2002). Musicoterapia. Recuperado de http://www.saludparati.com/musicoterapia

Soto, G. (2002). La música: un factor de evolución social y humana. Recuperado de http://iieh.com/autores/gsoto.html

Strayer, F. (1981). The nature and organization of altruistic behavior among preschool children. En J.P. Rushton y R.M. Sorrentino (Eds.), Altruism and helping behavior. Hillsdale: Lawrence Erlbaum.

\section{AUTORAS:}

\section{Ana María Botella Nicolás:}

Doctora en pedagogía por la Universitat de València. Es Licenciada en Geografía e Historia, especialidad Musicología y maestra en Educación Musical, por la Universidad de Oviedo. Grado profesional en la especialidad de piano. Durante el año 2001 obtiene por oposición plaza en el cuerpo de profesores de música de enseñanza secundaria en Alicante (actualmente en excedencia). Ha presentado diversas comunicaciones en jornadas y congresos sobre didáctica de la música así como distintas publicaciones. Es profesora contratada doctora del departamento de didáctica de la Expresión Musical, Plástica y Corporal de la Facultad de Magisterio de la Universitat de València. Forma parte de la Comisión de Coordinación Académica del Master Universitario en Profesor/a de enseñanza secundaria de la UVEG y del Máster de Investigación en didácticas específicas. Desde Febrero de 2015 dirige el aula de música del Vicerrectorado de Cultura e Igualdad.

Orcid: 0000-0001-5324-7152 y ResearchID: N-1375-2014. 


\section{Carolina Montesinos Boscá:}

Es Grado en Maestra en Educación Primaria, especialidad de Educación Musical por la Universitat de Valencia. Posee el Título profesional de música en la especialidad de Piano por el Conservatorio Superior de Música de Valencia. 